\title{
Use of perfusion index from pulse oximetry to determine efficacy of stellate ganglion block
}

\section{Hajime Yamazaki \\ Junichi Nishiyama \\ Toshiyasu Suzuki}

Department of Anesthesiology, Surgical Science, Tokai University

School of Medicine, Kanagawa, Japan
Correspondence: Hajime Yamazaki Department of Anesthesiology, Surgical Science, Tokai University School of Medicine, 143 Shimokasuya, Isehara, Kanagawa, 259-II43, Japan

$\mathrm{Tel}+8 \mid 46393$ II 2 I

Fax $+8 \mid 463919260$

Email y-hajime@is.icc.u-tokai.ac.jp
This article was published in the following Dove Press journal:

Local and Regional Anesthesia

12 March 2012

Number of times this article has been viewed

Background: Stellate ganglion block (SGB) is a widely used procedure for treatment of pain in the head and upper body, but the clinical signs used to verify the effectiveness of SGB can be ambiguous or variable in some patients. We observed the chronological changes in perfusion index (PI) from pulse oximetry to determine if these changes correlated with the clinical signs associated with an effective SGB. We hypothesized that PI could provide an easy method to assess the effectiveness of SGB.

Method: We compared the chronologies in PI on the treated and untreated sides of 21 patients in whom treatment by SGB was found to be effective. The SGB was performed by administering $6 \mathrm{~mL}$ of $1 \%$ mepivacaine. The effectiveness of the SGB was confirmed on the basis of clinical signs. Additionally, in two patients we tested whether increased PI on the treatment side correlated with increased microcirculation as measured by laser-Doppler blood flowmetry.

Results: On the side treated by SGB, PI increased $61.4 \%$ in the earlobe and $60.5 \%$ from baseline values in the upper limbs, at 5 minutes after initiation of the procedure. Differences in PI before and after treatment were significant at both sites. No time-course increases in PI were found on the untreated side at either site. Following SGB, increases in PI correlated with increases in blood flow as measured by laser-Doppler flowmetry.

Conclusion: PI increased in the earlobe and upper limbs on the treated side of 21 patients who received an effective SGB but not on the untreated side. The positive correlations between changes in PI and both presence of clinical signs and changes in blood flow in the skin microcirculation indicate a sympatholytic effect, suggesting that the PI could be useful in determination of the efficacy of SGB.

Keywords: stellate ganglion block, pulse oximetry, peripheral perfusion, perfusion index, skin microcirculation

\section{Introduction}

A stellate ganglion block (SGB) is defined as a nerve block which simultaneously blocks the cervical sympathetic trunks, the vertebral ganglia, the preganglionic and postganglionic fibers of the lower cervical sympathetic ganglia, and the upper thoracic sympathetic ganglia. SGB is mainly used for treatment of painful disorders of the head and neck, upper limbs, and upper chest. The presence of clinical signs such as Horner's sign, hypohidrosis and vasodilatation in the upper limbs are used to verify that SGB is effective in the clinical settings. However, these signs can be ambiguous in some patients. Perfusion index (PI), which is automatically calculated by pulse oximetry, provides an indication of peripheral perfusion at the sensor site. We examined the relationship between the presence of clinical signs and changes in PI after SGB to 
determine if PI could be used to verify the efficacy of SGB. Additionally, we verified that increased PI correlates with increased skin microcirculation (sympatholytic effect) by studying changes in PI and changes in blood flow as measured by laser-Doppler flowmetry following effective SGB.

\section{Methods}

Following our institutional ethics committee approval and obtaining written informed consent, a retrospective survey of patients scheduled for SGB between June and November 2006 was conducted. Exclusion criteria included patients receiving treatment for circulatory disturbances in the upper limbs, diabetes mellitus, or hypertension. For procedure, the ambient temperature was $24^{\circ} \mathrm{C}$ to $25^{\circ} \mathrm{C}$ and patients were put in a supine position. The pulse oximetry sensors (LNCS adult adhesive sensors connected to Masimo SET $^{\circledR}$ Radical $^{\mathrm{TM}}$ pulse oximeters; Masimo Corp, Irvine, CA) were affixed, bilaterally, to the patients' earlobes, and the tips of their thumbs. The pulse oximeters were connected to personal computers with data collection software (Physiolog TM; Masimo Corp) for the continuous recording of PI. The baseline PI value was recorded after 15 minutes of bed rest, before the SGB procedure. Blood pressure and pulse rate were measured from the upper arm on the opposite side of the SGB. The SGB was performed by using $6 \mathrm{~mL}$ of $1 \%$ mepivacaine (Carbocaine ${ }^{\circledR}$; Hospira Inc, Lake Forest, IL) according to the paravertebral approach, using the anterior tubercle of the 6th cervical transverse process as a marker. The efficacy of the SGB was determined on the basis of clinical signs by confirming the presence or absence of Horner's sign, flushing and hypohidrosis on the face, and vasodilatation and hypohidrosis in the upper limbs on the treated side, compared to the side opposite the SGB (untreated side). To evaluate the correlation between PI and blood flow after SGB, pulse oximeter sensors and laser-Doppler blood flow meter sensors (CSF-2000 ${ }^{\circledR}$; Cyber Firm Inc, Tokyo, Japan) were attached, side by side, on the earlobes on the side treated by SGB of two patients. The SGB was performed according to the procedure described above. PI and laser-Doppler blood flowmetry measurements were recorded on the same time axis using data collection software specific for each measurement (Physiolog ${ }^{\mathrm{TM}}$ for PI and Trend BASICTM; Cyber Firm Inc, Tokyo, Japan, for the blood flow meter). The mean \pm standard deviation for each measurement was calculated. The change in PI was calculated as ([post PI-pre PI]/pre PI) $\times 100$ and expressed as a percentage. Statistical analyses were performed using the one-way analysis of variance, a Bonferroni/Dunn posthoc test, and Spearman's rank correlation coefficient. The criterion for significant difference was $P<0.05$.

\section{Results}

SGB was performed on 30 patients within the study period. The efficacy of SGB was determined on the basis of the observation of Horner's sign, flushing and hypohidrosis on the face, and vasodilatation and hypohidrosis in the upper limbs; and the results showed that SGB was effective in 21 patients $(70 \%)$. The remaining nine patients showed the presence of Horner's sign but clinical signs in the upper limbs were absent (Table 1). In all patients, there was no significant difference in blood pressure or pulse rate before and after the SGB.

\section{PI changes in the earlobe following SGB}

In patients for whom SGB was effective, baseline values of PI in the earlobes ranged from 0.13 to 1.25 on both sides of the patient. On the side of the SGB, PI rose continuously during the 30 -minute observation period. At 5 minutes, PI increased by $61.4 \%$ from baseline $(P=0.042)$. No changes from baseline were observed on the untreated side (Figure 1).

\section{PI changes in upper limbs following SGB}

The baseline values of PI in the upper limbs ranged from 3.10 to 12.13 on both sides of the patient. On the side of the SGB, PI increased $60.5 \%$ from baseline in the first 5 minutes. Differences from baseline were significant $(P=0.008)$. At 15 minutes from SGB, PI reached a peak with an increase of $87.3 \%$ from baseline. On the side opposite the SGB there was a time-course decrease in PI from baseline but the difference was not significant (Figure 2).

Table I Patient diagnosis requiring treatment with SGB

\begin{tabular}{lll}
\hline & & $\mathbf{N}=\mathbf{2}$ I \\
\hline Diagnosis & Cervical spondylosis & I $3(6 \mathrm{I} .9 \%)$ \\
& Cervical disc hernia & I (4.8\%) \\
& Herpes zoster & $5(23.8 \%)$ \\
& Facial palsy & I (4.8\%) \\
& Sudden deafness & I (4.8\%) \\
Age (years) & & $54.4(34-7 \mathrm{I})$ \\
Gender (male:female) & & $\mathrm{I}: 10$ \\
Block side (R:L) & & $9: 12$ \\
\hline
\end{tabular}

Note: Data shown are number (percentage) or mean (range).

Abbreviation: SGB, stellate ganglion block. 


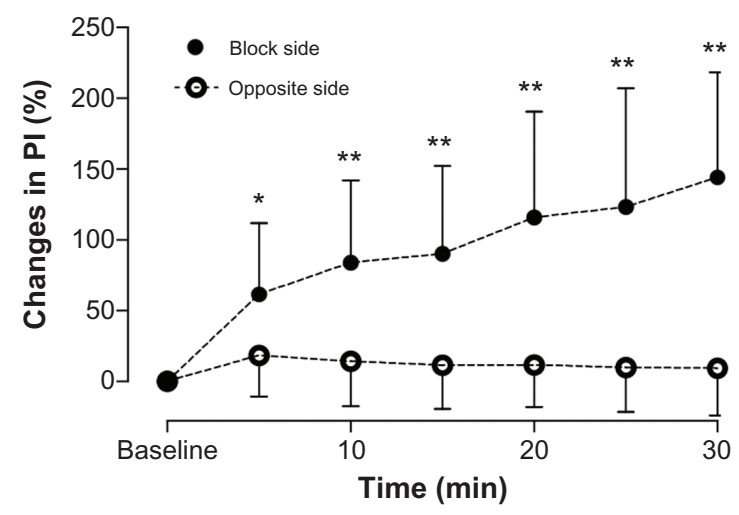

Figure I Chronological changes in PI in the earlobe. On the side of the SGB, $61.4 \% \pm 50.5 \%, 84.0 \% \pm 58.1 \%, 90.2 \% \pm 62.1 \%, 115.8 \% \pm 74.9 \%, 123.2 \% \pm 83.8 \%$, and $144.2 \% \pm 74.1 \%$ increases were found at each respective time. No chronological changes were found on the opposite side of the SGB.

Note: $* P<0.05$ vs baseline; $* * P<0.01$ vs baseline.

Abbreviations: SGB, stellate ganglion block; PI, perfusion index.

\section{Relationship between the PI and blood flow}

When blood flow in the skin microcirculation and PI was measured in two patients in whom SGB was effective as determined by the presence of Horner's sign and facial flushing, a positive linear correlation was found. In both patients, PI increased as blood flow increased at the sensor site (the earlobe). The correlation coefficient $\left(\mathrm{r}^{2}\right)$ was 0.89 in one patient and 0.68 in the other so some variability in the strength of the correlation existed between patients (Figure 3).

\section{Discussion}

Various techniques have been employed to verify the efficacy of SGB, most of which consist of evaluations based on skin temperature. ${ }^{1-8}$ However, the standard of care is to

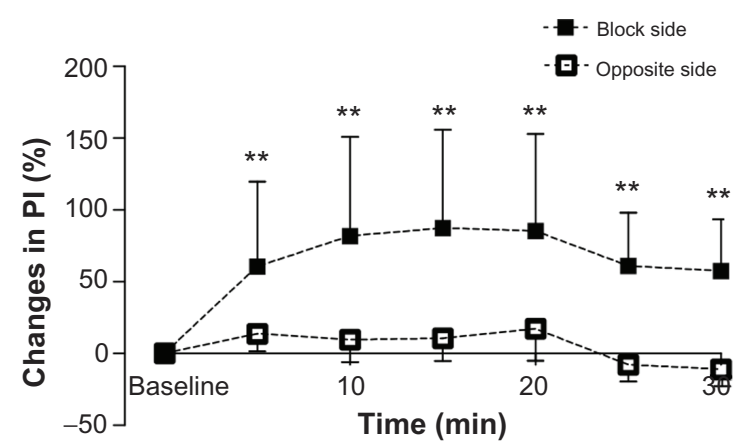

Figure 2 Chronological changes in PI in the upper limbs. On the side of the SGB, $60.5 \% \pm 59.3 \%, 81.9 \% \pm 68.9 \%, 87.3 \% \pm 68.6 \%, 85.4 \% \pm 67.5 \%, 61.0 \% \pm 37.0 \%$, and $57.6 \% \pm 35.9 \%$ increases were found at each respective time. No chronological changes were found on the opposite side of the SGB.

Note: $* * p<0.01$ vs baseline.

Abbreviations: SGB, stellate ganglion block; PI, perfusion index. determine the efficacy of SGB based on the presence of clinical signs on the face and the upper limbs following the procedure. These signs can be ambiguous in some patients and there are no well-defined criteria for them. The SGB procedure involves injection of local anesthetic through skin and deeper "sympathetic nerve" tissue. The course of muscles and the prevertebral fascia at the puncture site of the nerve block needle ${ }^{9}$ contribute to the spread of the administered drug solution. ${ }^{10,11}$ The drug solution spreads into the fascia of the longus colli, and blocks the preganglionic and postganglionic fibers connected to the stellate ganglion but this does not necessarily mean that the stellate ganglion itself is blocked. ${ }^{12}$ However, if the drug solution is injected into the longus capitis muscle instead, the sympatholytic effect cannot be obtained in the upper limbs. ${ }^{1-3,10,12-18}$ Therefore, with an ineffective SGB some but not all of the clinical signs of an effective block may be present. An alternative method of determining the efficacy of SGB that is not dependent on variable and sometimes ambiguous clinical signs may be helpful in the clinical setting.

PI is an indicator of the relative strength of the pulsatile signal from pulse oximetry and has been found to be a reliable indicator of peripheral perfusion. PI is calculated by dividing the pulsatile signal (AC) by the nonpulsatile signal (DC) times 100 , and is expressed as a percent ranging from $0.02 \%$ to $20 \%$. A higher PI value, therefore, indicates a stronger pulsatile signal and better peripheral circulation at the sensor site. Because PI is an indicator of peripheral blood flow, and peripheral blood flow in the upper limbs is a clinical sign of an effective block, increased PI on the side of the block may be a reliable means to determine the efficacy of SGB. We confirmed PI as an indicator of peripheral blood flow in SGB patients using laser-Doppler blood flowmetry in two patients who received an effective SGB. A laser-Doppler blood flow meter irradiates tissues with a single wavelength of light at $780 \mathrm{~nm}$ and converts the scattered light from the tissues into electrical signals to measure the blood flow in the skin microcirculation system. The skin microcirculation system is composed of arterioles, capillaries, and venules, constituting the upper horizontal plexus in the dermal papillae and the lower horizontal plexus under the skin. ${ }^{19}$ The permitted depth for the measurements by laser-Doppler blood flowmetry is up to $3 \mathrm{~mm}$. Following an effective SGB, blood flow in the target tissues increases. PI and blood flow have been reported to be highly correlated ${ }^{20}$ and our study confirmed this finding (Figure 3), but the absolute value of blood flow could not be calculated from the PI. 

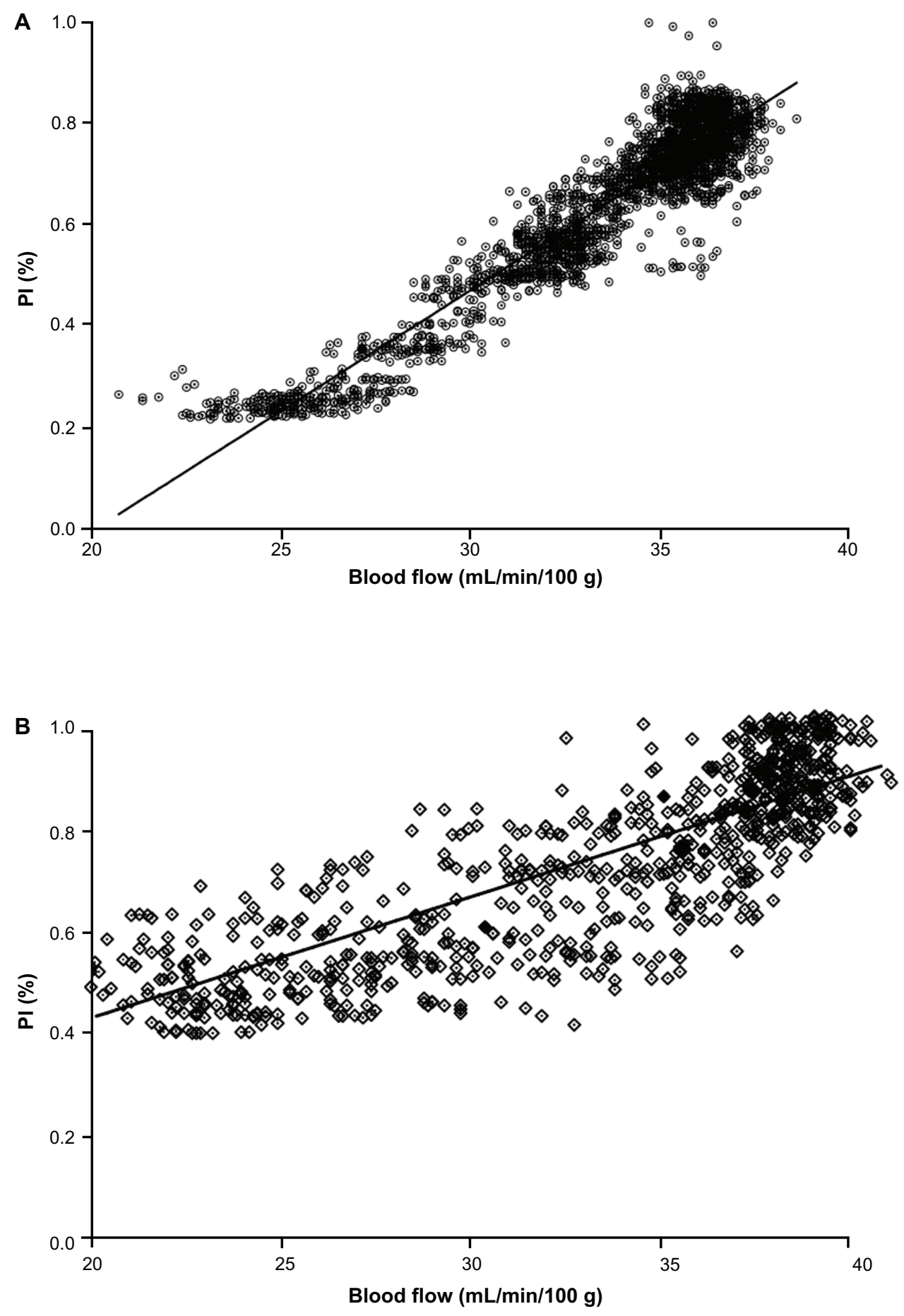

Figure 3 Relationship between PI and blood flow after SGB, as measured by pulse oximetry and laser-Doppler blood flow meter sensor attached to the earlobes of 2 patients. $(\mathbf{A}) \mathrm{PI}=0.047 \times($ blood flow $)-0.968, r^{2}=0.89,(B) P I=0.025 \times($ blood flow $)-0.053 \mathrm{I}, r^{2}=0.68$.

Abbreviations: SGB, stellate ganglion block; PI, perfusion index.

We investigated the impact of SGB on PI by conducting a study on 21 patients who were treated with SGB. We showed that there are predictable chronological changes in PI that occur with an effective SGB. In this study, clinical evaluations affirmed that SGB was effective in $70 \%(21 / 30)$ of the cases in which SGB was attempted. While PI did not increase on the side opposite the SGB, it increased both in the earlobe and in the upper limbs on the side of the SGB (Figure 4). The increase in PI on the side of the SGB (but not on the untreated side) suggests the increase in blood flow is caused by the sympatholytic effect of SGB and not due to reactions to local anesthetics or to other environmental factors (such as 


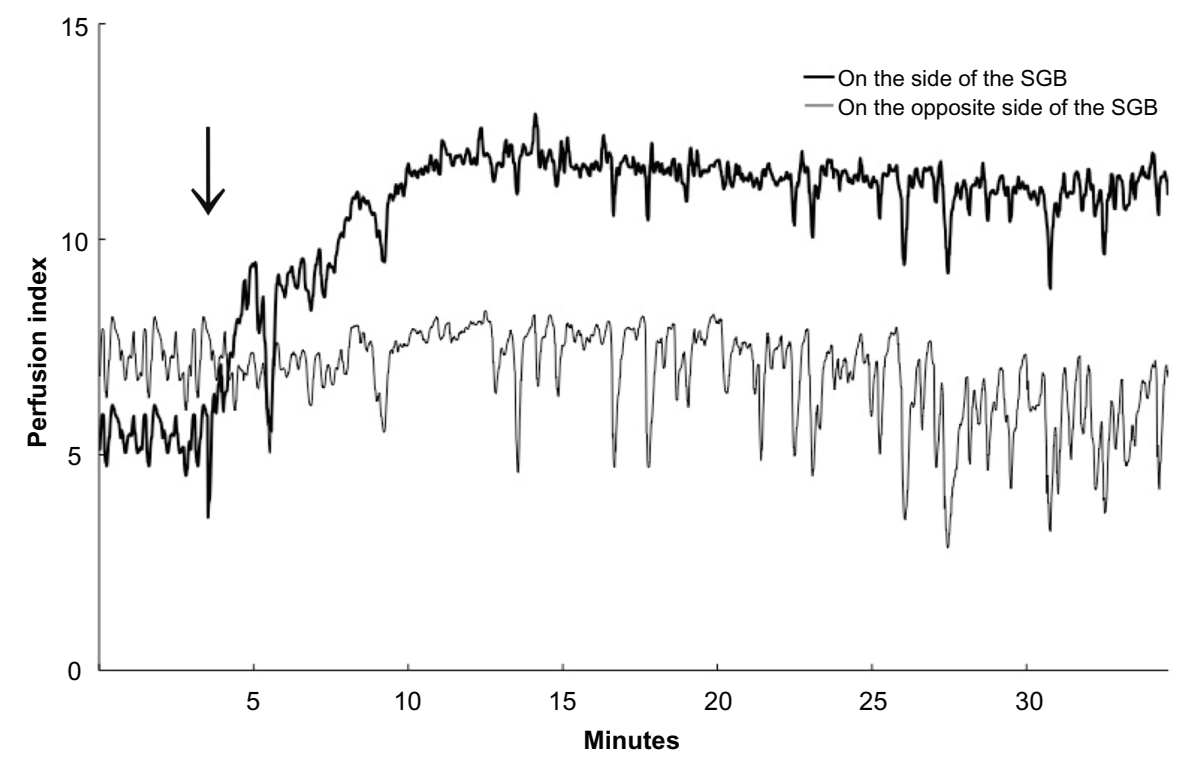

Figure 4 Chronological changes in PI in the upper limbs of a patient. The arrow indicates the time that SGB was performed. PI values on the side of the SGB increased chronologically, whereas those of the opposite side did not significantly following SGB. PI showed high frequency variations on both sides, but a time-course increase in PI was found only on the side of the SGB.

Abbreviations: SGB, stellate ganglion block; PI, perfusion index.

temperature or other stimuli). In a previous report, using an ultrasonic blood flow meter for measurement, Murakawa et al showed that the common carotid artery blood flow increased by $134.6 \% \pm 5.8 \%$ at 5 minutes, and by $179.7 \% \pm 11.1 \%$ at 20 minutes after performing SGB. ${ }^{21}$ In this study, we used laser-Doppler blood flowmetry to confirm changes in blood flow following SGB. When using PI to confirm the efficacy of a SGB, some factors should be taken into consideration. First, because body movements cause changes in the pulse oximetry AC/DC signal ratio ${ }^{22,23}$ before and after SGB the body posture of the patient should not be changed. The influence of the redistribution of body fluids, or changes in the way the sensor is positioned may affect the measurement of PI. Additionally, applying pressure to the sensor may cause changes in blood flow in the skin microcirculation, ${ }^{22-25}$ and therefore, should be avoided. In conclusion, we found that continuous measurements of the PI using a pulse oximeter could be useful for determining the efficacy of SGB.

\section{Disclosure}

The authors report no conflicts of interest in this work.

\section{References}

1. Matsumoto S. Thermographic assessments of the sympathetic blockade by stellate ganglion block (1) - Comparison between C7-SGB and C6-SGB in 40 patients. Masui. 1991;40(4):562-569.

2. Matsumoto S. Thermographic assessments of the sympathetic blockade by stellate ganglion block (2) - Comparison and analysis of thermographic patterns between C7-SGB and C6-SGB in 20 healthy volunteers. Masui. 1991;40(5):692-701.
3. Matsumoto S, Mitsuhata H, Hasegawa J. Thermographic assessments of the sympathetic blockade after stellate ganglion block (no 3) - the block at the base of the 6th cervical transverse process. Masui. 1992;41(1):111-118.

4. Benzon H, Avram MJ. Temperature increases after complete sympathetic blockade. Reg Anesth. 1986;11(1):27-30.

5. Malmqvist EL-Å, Bengtsson M, Sörensen J. Efficacy of stellate ganglion block: A clinical study with bupivacaine. Reg Anesth. 1992;17(6):340-347.

6. Murakawa K, Noma K, Matsuda M, et al. Changes of tympanic temperature by stellate ganglion block in facial palsy. Facial N Res JPN. 1997; 17:116-118.

7. Stevens RA, Storz A, Kao T-C, Powar M, Burgess S, Kleinman B. The relative increase in skin temperature after stellate ganglion block is predictive of a complete sympathectomy of the hand. Reg Anesth Pain Med. 1998;23(3):266-270.

8. Kapural L, Mekhail N. Assessment of sympathetic blocks. Tech Reg Anesth Pain Manage. 2001;5(3):82-87.

9. Ebraheim NA, Lu J, Yang H, Heck BE, Yeasting RA. Vulnerability of the sympathetic trunk during the anterior approach to the lower cervical spine. Spine (Phila Pa 1976). 2000;25(13):1603-1606.

10. Fukui T, Nakagawa G, Nishioka K, Fujioka Y, Okada Y. The study of anatomy of stellate ganglion in living human, and spread of injected solution by stellate ganglion block. Pain Clinic. 1993;14(2):226-231.

11. Shibata Y, Fujiwara Y, Komatsu T. A new approach of ultrasoundguided stellate ganglion block. Anesth Analg. 2007;105(2):550-551.

12. Wrete M. The anatomy of the sympathetic trunks in man. $J$ Anat. 1959;93(4):448-459.

13. Yamamuro M, Kaneko T. The comparison of stellate ganglion block at the transverse process of the 7th and the 6th cervical vertebra. Masui. 1978;27(4):376-389.

14. Yamamuro M, Eba K, Kaneko T, Yasuda T, Hitsuda H, Ono K. Stellate ganglion block at the sixth cervical vertebra (2). A study of technique. Pain Clinic. 1991;12(4):507-512.

15. Hogan QH, Erickson SJ, Haddox JD, Abram SE. The spread of solutions during stellate ganglion block. Reg Anesth. 1992;17(2):78-83.

16. Honma M, Murakami G, Sato TJ, Namiki A. Spread of injectate during C6 stellate ganglion block and fascial arrangement in the prevertebral region: an experimental study using donated cadavers. Reg Anesth Pain Med. 2000;25(6):573-583. 
17. Elias M. Cervical sympathetic and stellate ganglion blocks. Pain Physician. 2000;3(3):294-304.

18. Kaneko T, Date H, Yamamuro M. Spread of injectate during stellate ganglion block. Pain clinic. 2001;22(5):687-695.

19. Berardesca E, Lévêque J-L, Masson P. EEMCO guidance for the measurement of skin microcirculation. Skin Pharmacol Appl Skin Physiol. 2002; 15(6):442-456.

20. Ozaki M, Sessler D, Lopez M, Walter K. Pulse oximeter-based flow index correlates well with finger volume plethysmography. Anesthesiology. 1993;79(3):A542.

21. Murakawa K, Ishimoto E, Noma K, Ishida K, Nishijima M, Izumi R. Circulatory effects of stellate ganglion block in idiopathic facial palsy. Masui. 1994;43(3):356-360.
22. Vegfors M, Tryggvason B, Sjöberg F, Lennmarken C. Assessment of peripheral blood flow using a pulse oximeter. J Clin Monit. 1990;6(1):1-4.

23. Vegfors M, Lindberg L, Lennmarken C. The influence of changes in blood flow on the accuracy of pulse oximetry in humans. Acta Anaesthesiol Scand. 1992;36(4):346-349.

24. Tenland T, Salerud EG, Nilsson GE, Öberg PA. Spatial and temporal variations in human skin blood flow. Int J Microcirc Clin Exp. 1983;2(2):81-90.

25. Salerud EG, Tenland T, Nilsson GE, Öberg PA. Rhythmical variations in human skin blood flow. Int J Microcirc Clin Exp. 1983;2(2): $91-102$.

\section{Publish your work in this journal}

Local and Regional Anesthesia is an international, peer-reviewed, open access journal publishing on the development, pharmacology, delivery and targeting and clinical use of local and regional anesthetics and analgesics. The journal welcomes submitted papers covering original research, basic science, clinical studies, reviews \& evaluations, guidelines, expert opinion and commentary, case reports and extended reports. The manuscript management system is completely online and includes a very quick and fair peer-review system, which is all easy to use. Visit http://www.dovepress.com/testimonials.php to read real quotes from published authors. 